$U_{n}(\xi)$ as defined above. Since

$$
\sum_{k=0}^{N}\left(\begin{array}{l}
N \\
k
\end{array}\right) \xi^{k}(1-\xi)^{N-k}=[\xi+(1-\xi)]^{N}=1,
$$

it follows that

$$
U_{n}(\xi)=\sum_{k=n}^{N}\left(\begin{array}{l}
N \\
k
\end{array}\right) \xi^{n}(1-\xi)^{N-k} .
$$

On the other hand, by direct calculation, this is also the sum over all possible $k$ (from $n$ through $N$ ) of the probability of having the $n$th (and last) son on the $k$ th opportunity; so that

$$
U_{n}(\xi)=\sum_{k=n}^{N}\left(\begin{array}{l}
k-1 \\
n-1
\end{array}\right) \xi^{n}(1-\xi)^{k-n} .
$$

The first problem is to prove the identity of (C) and (D), for general $\xi$, without appeal to this probabilistic approach.

Among sterilized couples, there are, by our postulate, always exactly $n$ sons. In this sub-population, the expected number of daughters [a conditional expectation] is then

$$
\begin{aligned}
E[d] & =\sum_{k=n}^{N} \sum_{d=0}^{k-n} d\left(\begin{array}{c}
k-1 \\
n-1
\end{array}\right)\left(\begin{array}{c}
k-n \\
d
\end{array}\right) \xi^{n} \eta^{d}(1-\xi-\eta)^{k-n-d} / U_{n}(\xi) \\
& =\sum_{k=n}^{N}\left(\begin{array}{c}
k-1 \\
n-1
\end{array}\right) \xi^{n} \sum_{d=0}^{k-n} d\left(\begin{array}{c}
k-n \\
d
\end{array}\right) \eta^{d}(1-\xi-\eta)^{k-n-d} / U_{n}(\xi) \\
& =\sum_{k=n+1}^{N}\left(\begin{array}{c}
k-1 \\
n-1
\end{array}\right)(k-n) \xi^{n} \eta \sum_{d=1}^{k-n}\left(\begin{array}{c}
k-n-1 \\
d-1
\end{array}\right) \eta^{d-1}(1-\xi-\eta)^{k-n-d} / U_{n}(\xi) \\
& =n \frac{\eta}{\xi} \sum_{k=n+1}^{N}\left(\begin{array}{c}
k-1 \\
n
\end{array}\right) \xi^{n+1}[\eta+(1-\xi-\eta)]^{k-n-1} / U_{n}(\xi) \\
& =n \frac{\eta}{\xi} \sum_{k=n+1}^{N}\left(\begin{array}{c}
k-1 \\
n
\end{array}\right) \xi^{n+1}(1-\xi)^{k-n-1} / U_{n}(\xi)=n \frac{\eta}{\xi} U_{n+1}(\xi) / U_{n}(\xi),
\end{aligned}
$$

by (D). Thus, the ratio of the number of boys $(n)$ to the number of girls (which, by the strong law of large numbers, converges almost surely, i.e., with probability one, to $E[d])$ converges as $N$ tends to infinity to

$$
\frac{\xi}{\eta} V_{n}(\xi)=\frac{\xi}{\eta} U_{n}(\xi) / U_{n+1}(\xi)
$$

The second problem is to prove that this quantity increases with $n$, for any given $0<\xi<1$ and $\eta \neq 0$.

\title{
On the Roots of a Bessel Function Equation
}

Problem 86-8 by ANTHONy D. Rawlins (Brunel, The University of West London).

Show that the roots of the equation

$$
F(z) J_{\nu}(z)+z J_{\nu}^{\prime}(z)=0
$$


where $\operatorname{Im} F(z)<0$ and $\nu>-1$ (or $\nu=n$ an integer) can only lie in the second and fourth quadrant of the $z$-plane.

This problem arose from an investigation of a sound field radiated from an acoustically lined circular duct. It was necessary to show that only certain waves could propagate along the duct. These waves corresponded to the roots of the above equation when $F$ took a particular form.

\section{A Normalization Constant}

Problem 86-9 by Jerold R. Bottiger (U. S. Army Chemical Research and Development Center) and David K. CoHOon (Temple University).

We wish to predict the orientation of a small axisymmetric particle carrying a fixed electrical dipole moment $\vec{\mu}$ which is suspended in a gas with temperature $T$ and is in an electric field whose electric vector $\vec{E}$ is assumed to be in the direction of the positive $z$-axis of the laboratory coordinate system. We let $\theta$ be the angle between the electric field vector $\vec{E}$ and a vector $\vec{R}$ which is parallel to the axis of symmetry of the particle and we let $\alpha$ be the fixed angle between $\vec{R}$ and the dipole moment vector $\vec{\mu}$ which is fixed in a moving coordinate system whose axes are defined by poles and other points on the tumbling particle. The probability that the angle $\theta$ between the axis of symmetry of the particle and the electric vector $\vec{E}$ satisfies $\theta_{1}<\theta<\theta_{2}$ is

$$
\int_{\theta_{1}}^{\theta_{2}} P_{a}(\theta) d \theta=c \int_{\theta_{1}}^{\theta_{2}} \exp (b \cos (\alpha) \cos (\theta)) I_{0}(b \sin (\alpha) \sin (\theta)) \sin (\theta) d \theta
$$

where $c$ is a normalization constant, $b$ is directly proportional to the product of the lengths of the dipole moment and electric field vectors and is inversely proportional to temperature $T$ of the gas, and $I_{0}$ is the zeroth order modified Bessel function. Find $c$ by evaluating the right side of the equation

$$
\frac{1}{c}=\int_{0}^{\pi} \exp (b \cos (\alpha) \cos (\theta)) I_{0}(b \sin (\alpha) \sin (\theta)) \sin (\theta) d \theta,
$$

thereby determining the probability distribution $P_{a}(\theta)$.

\section{A Fourier Sine Series}

Problem 86-10 by M. L. Glasser (Clarkson College) and H. E. FetTis (Former Collaborating Problem Editor).

Find a closed form expression for the sum of the Fourier series

$$
\sum_{k=1}^{\infty}(-1)^{k}\left(k^{2}-\alpha^{2}\right)^{-1} \sin k x
$$

in terms of the hypergeometric function. Also, when $\alpha$ is rational, show that the sum can be expressed in elementary terms.

\section{SOLUTIONS}

\section{An Optimal Search}

Problem 63-9*, by RICHARD Bellman (The Rand Corporation).

Suppose that we know that a particle is located in the interval $(x, x+d x)$, somewhere along the real line $-\infty<x<\infty$ with a probability density function $g(x)$. We 